\title{
Algorithms and selective attention
}

\author{
PETER DIXON \\ Bell Laboratories, Murray Hill, New Jersey 07974
}

\begin{abstract}
Selective attention is hypothesized to be controlled by intentionally chosen algorithms that specify how stimuli are to be processed. These experiments attempted to measure the time to select such algorithms. Subjects were given a two-choice reaction time task with a variety of different possible stimuli. Prior to each trial, subjects viewed a cue under their control that indicated the two possible stimuli for the upcoming trial. During this interval, subjects presumably selected a stimulus identification algorithm appropriate for that pair of letters; the duration of the interval was used as an index of algorithm selection time. The results indicated that selection time increases with the number of alternative algorithms and that algorithms may consist, in part, of perceptual tests to determine which of the two possible stimuli was presented. A specific model of the selection process was proposed to account for the results.
\end{abstract}

A person's ability to attend to some aspects of the environment and not to others is determined both by what he intends to do and what he is able to do. On one hand, a person has a great deal of control over how incoming stimuli are processed. For instance, under many circumstances one can attend to one auditory channel rather than to another, or one can process only a single aspect of a multidimensional stimulus. On the other hand, one's intention can be limited by selective attention capacity. Thus, one cannot separate two auditory messages if they come from the same location and in the same voice (e.g., Treisman, 1964), and one cannot attend to only one of two integral stimulus dimensions (Garner \& Felfoldy, 1970). Thus, selective attention must be thought of as having both flexible, intentional components and relatively inflexible capacity limitations.

The flexible, intentional control of attention requires some sort of internal representation to indicate how the stimuli will be processed. This representation will be referred to as a selective attention algorithm. Presumably, it would indicate symbolically the sequence of operations needed to analyze the stimuli. The following assumptions will be made about how such an algorithm might be used: (1) It will be assumed that selective attention algorithms are determined prior to actually performing the task. Thus, the process of algorithm selection or construction can be separated temporally from the process of algorithm execution. (2) It will be assumed further that the internal representation of an algorithm entirely specifies the processing to be performed. This processing may include tests and operations that are conditional upon the stimuli actually

Portions of this research were reported at the 1979 Psychonomic Society meetings in Phoenix. The author wishes to thank Marcel Just and Susan Dumais for comments on previous drafts. Requests for reprints should be sent to Peter Dixon, Bell Laboratories, Murray Hill, New Jersey 07974. presented, but no other decisions about how to do the task need to be made after the algorithm has been chosen. (3) Finally, people can be assumed to be fairly adept at selecting an algorithm appropriate for a particular situation. Once someone understands the task demands, he should have little trouble in arranging his internal processing to produce the correct outcome. This property distinguishes algorithms from heuristic strategies, in that heuristics are generally not guaranteed to be successful.

These assumptions enable one to study the process of algorithm selection. They seem to be fairly plausible, and they will gain additional support to the extent that they produce reasonable and consistent results.

A selective attention algorithm should be distinguished from selective attention capacity. Attention capacity refers to what processes and operations people have at their disposal to analyze and respond to the stimulus. An attention algorithm refers to how a person intends to use those capacities. This can be illustrated by considering a classification task in which the stimulus can be a circle, a square, two circles, or two squares. Subjects can be asked to respond on the basis of number (e.g., press a button if two objects are shown), form (press a button if squares are shown), or both (press a button if one circle or two squares are shown). Although all three tasks map two stimuli onto each response, the two-dimensional task is much harder than either of the one-dimensional tasks (Fitts \& Biederman, 1965; Morin, Forrin, \& Archer, 1961). One interpretation of this finding is that people cannot process both number and form simultaneously. Thus, these results suggest a limitation of selective attention capacity.

However, the task also reveals a flexible, intentional aspect to attention. Although subjects cannot process both dimensions simultaneously, they can attend to either one by itself. Moreover, whether subjects attend to form or number is entirely under their own control 
and can be changed at will. In the present analysis, this is done by selecting an algorithm that specifies either the processing of form information or the processing of number information. One can conjecture about the nature of this processing. For instance, the form algorithm might operate by producing "circle" or "square" name codes to mediate the response (Posner \& Mitchell, 1967). Similarly, an algorithm to analyze number information might output an abstract number code with which the subject could make a numerical classification (Dixon, 1978; Shepard, Kilpatric, \& Cunningham, 1975). In either case, the algorithm that indicates how the stimuli are to be analyzed must be intentionally chosen by the subject prior to his actually performing the task.

The concept of selective attention algorithms proposed here is similar to other concepts proposed to account for flexible stimulus processing. For instance, Treisman (1969) has hypothesized that in an attention task, subjects make a variety of decisions about how incoming stimulation should be processed. These include decisions about the source of sensory input, how particular dimensions or stimulus properties should be analyzed, and what specific perceptual tests should be performed on those dimensions. Similarly, Egeth (1967) has suggested that instructions and motivation determine a stimulus coding strategy that subjects use to operate on the sensory input. Both of these constructs are intended to allow for flexibility in processing in the same way as a selective attention algorithm does.

The experiments reported in this paper measured the time it takes to choose selective attention algorithms in a letter identification task. The hope was that these algorithm selection time measurements would provide some insight into how algorithms are organized internally and how the selection process operates in this situation. Experiment 1 looked at whether selection time was affected by the number of alternative algorithms. If algorithm selection is analogous to other kinds of choice behavior, selection time may increase with the uncertainty about appropriate algorithm choices. Experiment 2 used selection time to investigate how algorithms are organized internally. The results of both experiments together suggest a specific model of algorithms and algorithm selection. Although Experiments 1 and 2 consider algorithm selection in only one particular task, it is possible that the process of algorithm selection is substantially the same in a variety of situations. Thus, the proposed model of algorithm selection may have fairly wide applicability.

The paradigm used in these experiments allows for a relatively direct measurement of algorithm selection time. Each trial was a choice reaction time trial with two possible stimulus letters and two possible responses. The possible letters varied from trial to trial. Prior to each trial, the subject was shown a cue indicating what the two possibilities were. On the basis of this information, the subject had to decide what sort of analysis and perceptual tests should be used to distinguish the two letters. In other words, the subject had to select an algorithm with which to analyze the stimulus display. Algorithm selection time was studied by looking at trial initiation time: the length of time subjects took to study the cue before starting the trial. When subjects had completed the algorithm selection process, they initiated the stimulus presentation, viewed the stimulus for that trial, and then responded as quickly as possible. Thus, two measures of performance were obtained on each trial: initiation time, which reflected the time to select an algorithm, and response time, which measured the time to execute the algorithm.

\section{EXPERIMENT 1}

One constraint that may operate on the selection of stimulus identification algorithms is the information transmitted by the choice of a particular algorithm. Behavior in a wide variety of situations has been shown to be a function of information and information-like measures (e.g., Fitts, 1954; Garner, 1962; Hick, 1952). It would also seem relatively easy to construct models of algorithm selection that predict a linear increase in algorithm selection time with the amount of information. For instance, one might hypothesize that in order to select an algorithm, subjects make a series of timeconsuming binary decisions. If each of these decisions reduced the number of alternative algorithms in half, the time to select a single algorithm would be linear in the logarithm of the number of alternatives, that is, the information content of that choice (cf. Hick, 1952).

Experiment 1 investigated the effect on algorithm selection time of varying the number of alternative algorithms. Algorithms were manipulated by changing the stimuli that were possible in a choice reaction time task. It was assumed that a different algorithm would be used for each different set of possible stimuli. The number of possible algorithms was varied across conditions. In the two-alternative condition, there were two possible algorithms (i.e., two possible pairs of stimuli), in the four-alternative condition, there were four possible algorithms, and in the eight-alternative condition, there were eight. Algorithm selection time was then measured as a function of the number of alternative algorithms.

\section{Method}

The subjects' basic task was a choice reaction time task with two possible stimuli and two possible responses. The stimuli were capital letters presented on a Minibee- 100 computer terminal under the control of a PDP-11/34 timesharing system. The responses consisted of pressing one of two buttons, on which the subject rested his index fingers. On each trial, a cue consisting of a pair of letters indicated which letter went with the left and right buttons. For instance, if the cue was " $\mathrm{X} \mathrm{G}$," the subject was to press the left button if an " $X$ " was shown and the right button if a "G" was shown.

Figure 1 shows the sequence of events on a trial. The computer displayed a fixation pattern consisting of two asterisks. The subject then pressed a foot pedal to view the cue for that trial. After $500 \mathrm{msec}$, the asterisks were replaced by the letter 


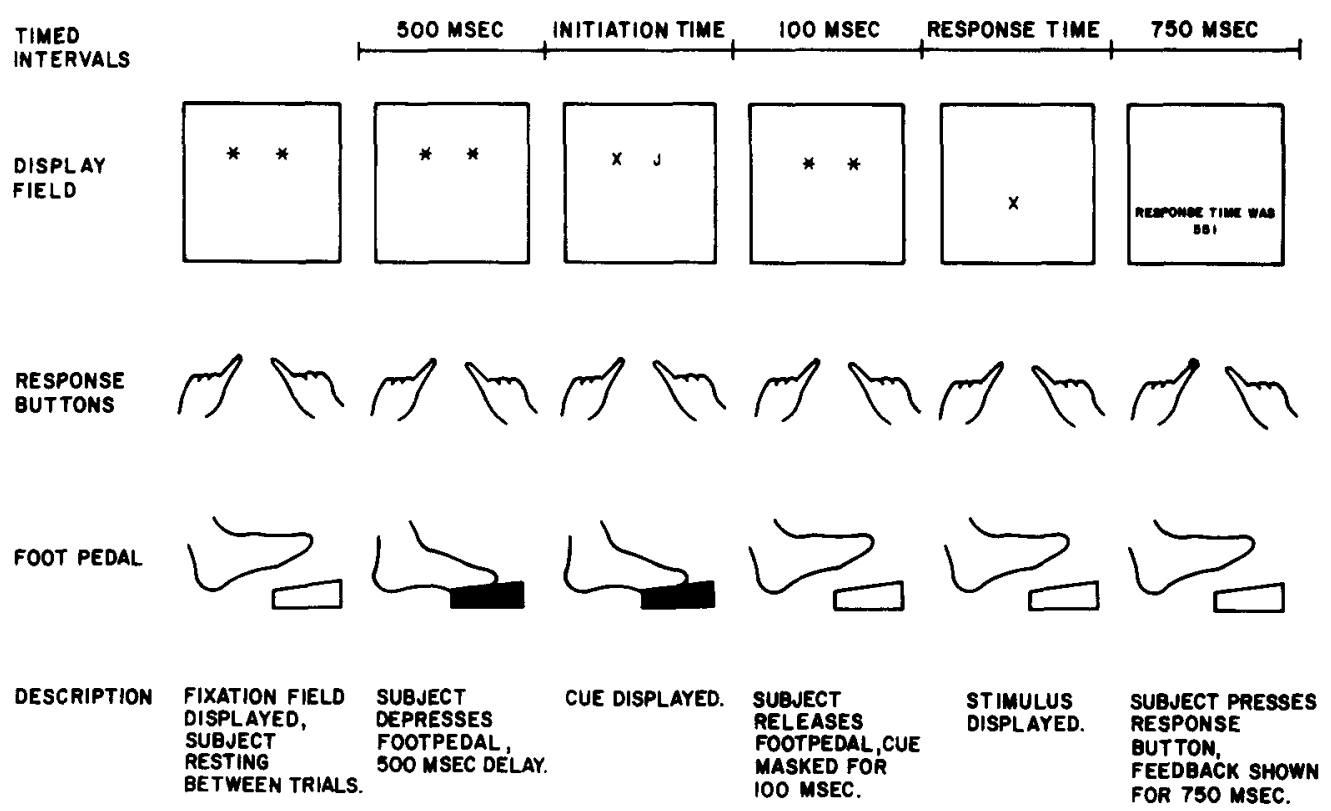

Figure 1. Sequence of events on each trial in Experiment 1.

pair cue. When the subject released the foot pedal, the cue was masked for $100 \mathrm{msec}$ by another pair of asterisks. One of the two letters in the cue was then presented, centered just below the position in which the cue had been. The subject's response terminated the display. Response time feedback was shown for $750 \mathrm{msec}$ if the subject responded correctly, and the message "incorrect" was shown for $1,250 \mathrm{msec}$ if the subject pressed the wrong button. Two times were measured on each trial. Trial initiation time was measured from the onset of the cue to the release of the foot pedal, and response time was measured from the onset of the stimulus to the buttonpress response. At a typical viewing distance of $50 \mathrm{~cm}$, a letter space subtended a visual angle of $.5 \mathrm{deg}$. Thus, the cue subtended about $1.5 \mathrm{deg}$ horizontally. The vertical separation of the cue position and the stimulus position was about $1.2 \mathrm{deg}$.

A different set of eight letter pairs was constructed for each subject by randomly dividing a set of 16 letters consisting of all the consonants except N, P, V, and W. Each subject participated in a condition with two possible letter pairs, a condition with four possible letter pairs, and a condition with eight possible letter pairs. The letters in each pair were always shown in the same left-to-right order. Letter Pairs 1 and 2 were used in the two-alternative condition, Pairs 1-4 were used in the fouralternative condition, and all eight pairs were used in the eightalternative condition. Thus, Letter Pairs 1 and 2 occurred in all three conditions. Each condition consisted of 10 practice trials, followed by 32 test trials in which all possible cues and stimuli occurred equally often. Error trials were rerun at random points during the condition.

Two subjects were tested with each of the six possible orders of the three conditions, for a total of 12 subjects. The conditions were administered first in the assigned order and then in the reverse order, for a total of six blocks of trials. Subjects were told that they were being timed on both initiation time and response time, but that it was important only to minimize response time. In addition, it was suggested that they could shorten their response time by taking longer to prepare for each trial. Subjects were informed about the cues that would occur in each condition. Undergraduates at Carnegie-Mellon University served as subjects in partial fulfillment of course requirements. One subject was replaced because of initiation times twice as long as those of any other subject.

\section{Results}

As shown in Figure 2, initiation time increased linearly across conditions $[F(1,66)=19.72, p<.001]$. The residual was not significant $[F(1,66)<1]$. The linear trend for response time was not significant $[F(1,66)=3.22]$. However, there was a significant quadratic trend $[F(1,66)=9.37, p<.005]$, reflecting the $18-\mathrm{msec}$ increase in response time from two to four alternatives. The analysis of initiation and response times was performed on the means for correct trials for each subject, condition, and letter pair. The trends across conditions were assessed with planned orthogonal contrasts on Letter Pairs 1 and 2 only, to avoid confounding the effects of condition with the effects of particular stimuli. A small percentage of the data points (1.1\% of the initiation times and $.7 \%$ of the response times) were identified as outliers more than 3 standard deviations greater than their respective means. These times were discarded and the means were recalculated before the analyses were performed. Error rates are shown in Table 1 .

\section{Discussion}

These results clearly show that algorithm selection time was affected by the number of alternative algorithms. More interestingly, selection time was apparently linear in the logarithm of the number of alternatives, suggesting that the information content of the algorithm choice is a determinant of selection time. This seems to be a plausible and parsimonious interpretation of the results, since information measures have been found to be important in a wide range of situations, such as motor control (Fitts, 1954), discrimination judgments (Garner, 1962), and reaction time (Hick, 1952). How- 


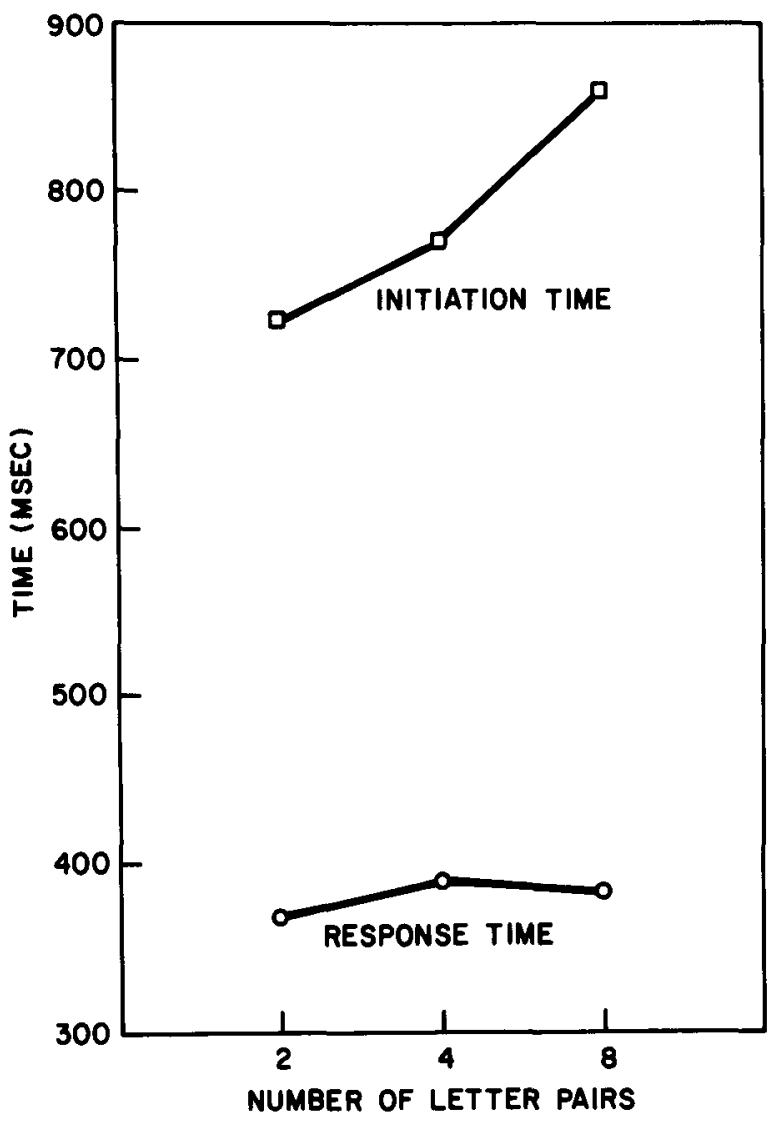

Figure 2. Mean response and initiation times for Letter Pairs 1 and 2 on correct trials in Experiment 1.

Table 1

Mean Times and Error Rates in Experiment 1

\begin{tabular}{cccc}
\hline Pairs & $\begin{array}{c}\text { Initiation } \\
\text { Time }\end{array}$ & $\begin{array}{c}\text { Response } \\
\text { Time }\end{array}$ & $\begin{array}{c}\text { Percent } \\
\text { Error }\end{array}$ \\
\hline \multicolumn{5}{c}{$1-2$} & \multicolumn{2}{c}{ Two-Alternative Condition } \\
& 724 & 370 \\
Four-Alternative Condition & 9.7 \\
$1-2$ & 770 & 388 & \\
$2-4$ & 758 & 393 & 10.3 \\
& Eight-Alternative Condition & \\
$1-2$ & 858 & 383 & 7.2 \\
$2-4$ & 855 & 401 & 11.5 \\
$4-8$ & 849 & 396 & 9.4 \\
\hline
\end{tabular}

ever, this conclusion should be made with caution, since the results are also statistically consistent with other functional relationships. Even though there was no significant deviation from the log-linear trend, the results actually lie between a purely linear trend and a log-linear one. The variability of the initiation times prevents one from specifying with certainty what the functional relationship actually is.

The algorithms used in this task may differ in terms of how attention is allocated to different letter features. Each algorithm could specify one feature that differentiates the two possible stimuli and then link it to the appropriate response. For instance, if the two possible letters were " $B$ " and " $R$," the subject might decide to attend to the lower right-hand portion of the stimulus to detect the presence or absence of the tail on the "R." If this analysis is correct, algorithm selection time would be related more to the number of possible distinguishing letter features than to simply the number of possible letter pairs. Further evidence on this kind of model will be considered in Experiment 2.

Response time in this experiment increased relatively little across conditions, even though there was a large increase in initiation time. This would be expected if subjects had completed the selection process prior to releasing the foot pedal and starting the response time interval. That is, any given algorithm should take the same amount of time to execute in all conditions, regardless of the number of alternatives from which it was selected. The small increase in response time from two to four alternatives can plausibly be attributed to initiation time "error." Subjects may have sometimes released the foot pedal too soon and completed the process of algorithm selection during the response interval. Note that this kind of error would also result in shorter initiation times. Thus, it is not surprising that the response time effect found in this experiment corresponded to a small deviation in initiation time from a linear fit.

It is useful to consider what might have happened if subjects had ignored the cue. In that case, subjects would have had to remember several stimuli for each response. For instance, if the two cues in the twoalternative condition had been " $\mathrm{J} X$ " and " $F \mathrm{Q}$," subjects would have had to remember that "J" and " $F$ " were both associated with the left button and that "X" and " $Q$ " were both associated with the right button. Similarly, in the four- and eight-alternative conditions, there would have been four and eight stimuli, respectively, for each response. Choice reaction time tasks that vary the number of possible stimuli for each response show that an increase in response time tends to occur for each additional stimulus (e.g., Fitts \& Biederman, 1965). In general, these studies suggest that response time might increase by $50-100 \mathrm{msec}$ for each bit of stimulus information. Thus, if the cue had been completely ignored in Experiment 1, response time could have been expected to increase by $100-200 \mathrm{msec}$ across the three conditions, but initiation time would have been constant. The fact that response time was instead relatively unchanged across conditions implies that some useful preparation must have occurred during the trial initiation interval.

An alternative explanation for the results of Experiment 1 might be based on the idea of cue uncertainty. Since the number of cues increased across conditions in the same way as did the number of algorithms, it might be argued that the time to encode the information in the cue increased with the amount of uncertainty about which cue would be presented. In order to test this 
hypothesis, 12 additional subjects viewed letter pairs under conditions identical to those of Experiment 1. The task in this case was to decide whether a letter presented just prior to the pair was one of the two shown. The number of letter pairs in a block of trials was two, four, or eight, as in Experiment 1. The prediction was that if initiation time increased because of encoding processes, response time in this task would show the same effect. However, the total range of response times for both positive and negative responses across the three conditions was less than $10 \mathrm{msec}$. Thus, cue encoding time does not appear to play a role in the present initiation time results.

\section{EXPERIMENT 2}

A stimulus identification algorithm might be organized in several different ways. As mentioned previously, one possibility is that it consists of tests performed on particular letter features designed to distinguish which of the two possible letters was shown. Different algorithms would then have to be used for different pairs of letters. Another possibility is that an algorithm consists of two independent mechanisms, each of which is responsible for identifying one of the two possible stimuli. One such proposal is that an algorithm consists of two independent stimulus-response links. Each mechanism would be specific to a particular letter and would initiate the response associated with that letter. Each time two different letters are used on a trial, two new mechanisms would have to be selected for the algorithm. This view of an algorithm makes the same prediction for the results of Experiment 1 as does the assumption that algorithms are specific to letter pairs.

One way to distinguish between these two possibilities is to vary the number of different letter pairs while leaving the total number of letters unchanged. If algorithm selection time increases under such circumstances, it would indicate that algorithms are specific to pairs of letters, not to the individual letters themselves. This would be consistent with the hypothesis that the stimulus identification algorithms consist of tests to distinguish two letters, rather than two independent mechanisms to identify individual letters.

Experiment 2 manipulated the number of possible letter pairs by varying how the stimuli were paired with each other to form the cues. One condition was the same as the two-alternative condition in Experiment 1. There were four possible stimuli ("X," "J," "F," and "Q"), and each letter was paired with only one other letter, resulting in two possible pairs (e.g., "X J" and "F Q"). In another condition, two other algorithms were obtained by switching the letters in the two pairs. Instead of " $X$ " always appearing with "J," it could sometimes occur with "Q," and instead of "F" always occurring with "Q," it could sometimes occur with "J." The pairs "X Q" and "F J" could then be added to the pairs
" $\mathrm{X} \mathrm{J}$ " and "F Q," for a total of four pairs. This manipulation of pairing doubled the number of letter pairs without increasing the number of letters.

In addition to varying letter pairing, Experiment 2 also varied the stimulus-response mapping. For instance, if "J X" was one cue, another might be "X J." The same two letters would be used in both algorithms, but the responses associated with them would be reversed. In a similar paradigm, Dixon and Just (Note 1) found that uncertainty about the order of the letters in the cue produced a substantial increase in trial initiation time. They referred to this result as a "laterality" effect, since it involved an assignment of side (left or right hand) to stimuli. Experiment 2 attempted to replicate the laterality effect and to study its relationship to the effect of varying letter pairing.

\section{Method}

The task, procedure, and apparatus were generally the same as those in Experiment 1 , with the exception that there was a 250-msec blank interval between the cue and the stimulus, rather than a $100-\mathrm{msec}$ mask. Four conditions were used: fixed, variable pairing, variable laterality, and variable pairing/ laterality. In the fixed condition, only two letter pairs were shown. The pairs were chosen randomly for each subject from the letters " $F$," "X," " $F$," and " $Q$." In the variable pairing condition, the letters were paired in two different ways. For instance, if the two cues in the fixed condition were " $\mathrm{X}$ " and "F $Q$," the four cues in the variable pairing condition were "X J," "F Q," "X Q," and " $F J . "$ In the variable laterality condition, letter pairs always remained the same, but they were shown in reverse order half of the time. Thus, " $\mathrm{X}$ " and " $\mathrm{X} J$ " might both be possible cues. In the variable pairing/laterality condition, each letter could be paired with two other letters and could appear on either side. Thus, there were eight possible cues in this condition. Exemplary letter pairs for each of the conditions are shown in Table 2 .

Each condition consisted of a block of 48 trials in which all possible cues and stimuli occurred equally often in a random order. Sixteen randomly chosen practice trials preceded each block. Subjects participated in the four conditions once; the order of the conditions was balanced across subjects so that each condition occurred in each serial position during the session an equal number of times. Twelve undergraduates at CarnegieMellon University served as subjects, in partial fulfillment of course requirements.

Table 2

Sample Cues in Experiment 2

\begin{tabular}{cccc}
\hline & \multicolumn{2}{c}{ Condition } \\
\hline & & & \\
Fixed & Variable & $\begin{array}{c}\text { Variable } \\
\text { Laterality }\end{array}$ & $\begin{array}{c}\text { Variable } \\
\text { Pairing/ } \\
\text { Laterality }\end{array}$ \\
\hline XJ & XJ & XJ & XJ \\
FQ & FQ & FQ & FQ \\
& XQ & JX & XQ \\
& FJ & QF & FJ \\
& & & JX \\
& & & QF \\
& & & QX \\
& & & JF \\
\hline
\end{tabular}




\section{Results}

As is shown in Figure 3, initiation time increased when letter pairing varied $[F(1,120)=9.75, p<.005]$. The effect on initiation time of varying laterality did not reach significance $[F(1,120)=3.57, .05<p<.10]$. However, a reliable laterality effect was found for response time $[F(1,120)=9.84, p<.005]$. This pattern of results suggests that subjects sometimes traded an initiation time increase in the conditions with variable laterality for an increase in response time, perhaps by initiating the stimulus presentation before completing the process of algorithm selection. As noted previously, this kind of tradeoff might be referred to as initiation time "error." If subjects had minimized their response time uniformly across conditions, a significant laterality effect on algorithm selection time might have been observed. The interaction of pairing and laterality did not approach significance for either response or initiation time. The analyses were performed on the mean response and initiation times for each subject, condition, and letter pair, excluding outliers greater than 3 standard deviations $(2.2 \%$ of the initiation times and

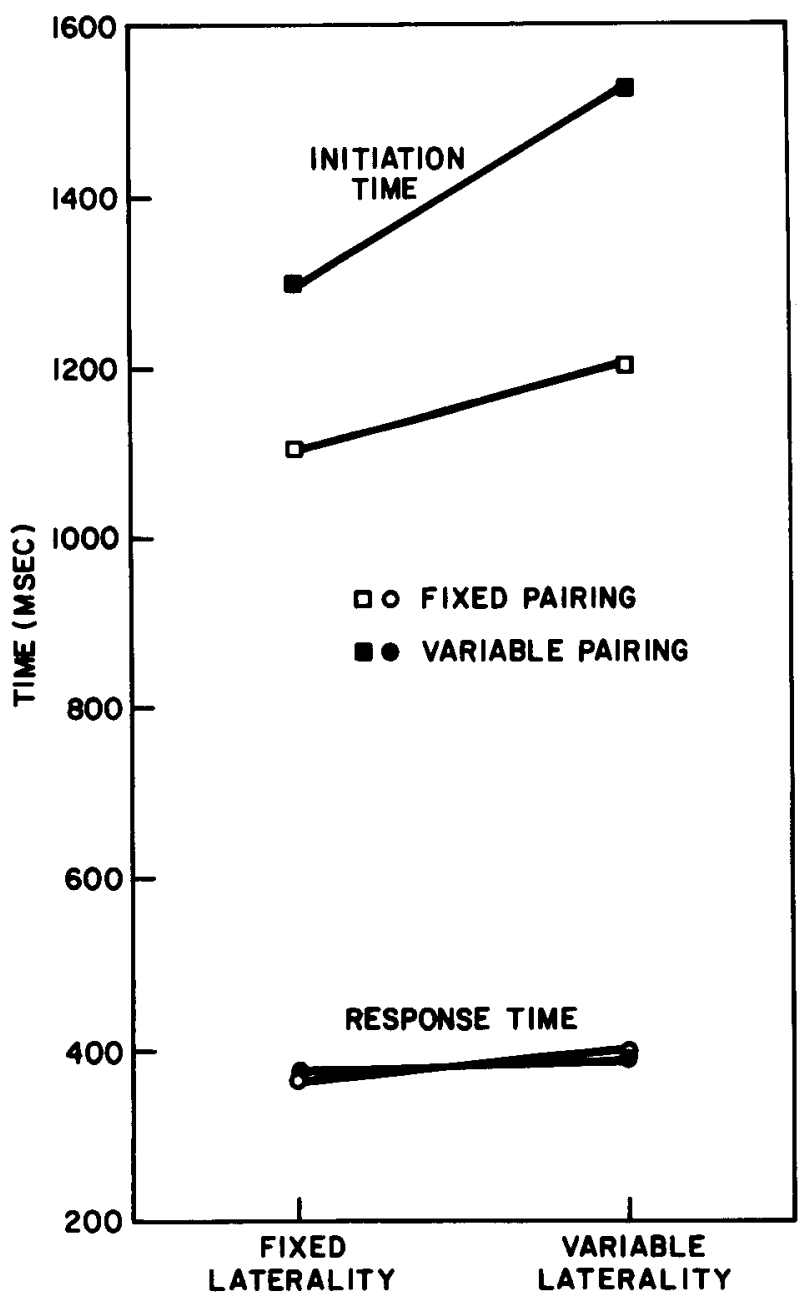

Figure 3. Mean response and initiation times for Letter Pairs 1 and 2 on correct trials in Experiment 2.
Table 3

Mean Times and Error Rates in Experiment 2

\begin{tabular}{|c|c|c|c|}
\hline Pairs & $\begin{array}{c}\text { Initiation } \\
\text { Time }\end{array}$ & $\begin{array}{c}\text { Response } \\
\text { Time }\end{array}$ & $\begin{array}{c}\text { Percent } \\
\text { Error }\end{array}$ \\
\hline \multicolumn{4}{|c|}{ Fixed Condition } \\
\hline $1-2$ & 1108 & 371 & 5.0 \\
\hline \multicolumn{4}{|c|}{ Variable Pairing Condition } \\
\hline $\begin{array}{l}1-2 \\
3-4\end{array}$ & $\begin{array}{l}1204 \\
1239\end{array}$ & $\begin{array}{l}397 \\
379\end{array}$ & $\begin{array}{l}4.2 \\
3.8\end{array}$ \\
\hline \multicolumn{4}{|c|}{ Variable Laterality Condition } \\
\hline $\begin{array}{l}1-2 \\
5-6\end{array}$ & $\begin{array}{l}1297 \\
1294\end{array}$ & $\begin{array}{l}378 \\
401\end{array}$ & $\begin{array}{l}3.5 \\
5.6\end{array}$ \\
\hline \multicolumn{4}{|c|}{ Variable Pairing/Laterality Condition } \\
\hline $\begin{array}{l}1-2 \\
3-4 \\
5-6 \\
7-8\end{array}$ & $\begin{array}{l}1531 \\
1584 \\
1650 \\
1538\end{array}$ & $\begin{array}{l}389 \\
379 \\
382 \\
394\end{array}$ & $\begin{array}{l}5.6 \\
9.0 \\
8.3 \\
4.2\end{array}$ \\
\hline
\end{tabular}

$.9 \%$ of the response times). The effects of interest were assessed with planned orthogonal contrasts on data for letter pairs that occurred in all four conditions. Errors are shown in Table 3.

\section{Discussion}

The results show that initiation time increases as a function of the number of letter pairs, even when the number of letters does not change across conditions. This suggests that an algorithm is specific to pairs of letters rather than to individual letters, and that it may consist of tests to distinguish the two letters in the pair. It also adds weight to the conclusion that subjects selected an appropriate algorithm during the initiation time interval and that it was this process that differentially affected initiation time.

The algorithms used in these experiments are probably not independent of the required response. Consider what might have happened if the response had been to name the stimulus letter. Uncertainty about possible stimuli has little effect on the naming of familiar stimuli (e.g., Brainard, Irby, Fitts, \& Alluisi, 1962), suggesting that if the present experiments had been run with a naming task, no effects would have been found for either initiation time or response time. The conclusion from such a result would be that a single letter-naming algorithm was used in all of the conditions and that no trial-by-trial algorithm selection was necessary. This letter-naming algorithm may be very different from the ones used for pressing buttons. For instance, it may produce a phonemic code that would be appropriate only for making a vocal response and inappropriate for deciding which of two buttons to press. On the other hand, an algorithm that detects distinguishing letter features may not be much help in finding a letter's name. Thus, algorithms for analyzing stimuli may take into account the required response by producing an internal code that is appropriate for that response.

An effect of laterality on initiation time would be consistent with the idea that attention algorithms produce codes tailored to particular responses. Although 
only weak evidence for a laterality effect was found in Experiment 2, the results suggest that a new algorithm has to be selected whenever the stimulus-response mapping is changed. This may be because a given algorithm produces an internal code appropriate for only one response, and when a new response is required (e.g., pressing the right button instead of the left) a different algorithm must be used. This view suggests that it may be difficult to separate stimulus identification processes from response selection processes.

\section{GENERAL DISCUSSION}

The results of these experiments lead to several conclusions about algorithm selection in tasks of this type. The first is that the selection process can be manipulated and measured. This is an important empirical validation of the idea that selective attention entails the use of algorithms that are actively chosen for a given situation. Second, the results of Experiment 1 indicate that the time to make such a selection increases with the uncertainty about the algorithm appropriate for a given trial; the more alternatives there are, the longer the algorithm selection time is. One possibility consistent with the results of Experiment 1 is that algorithm selection time is a linear function of the information content of the chosen algorithm. Finally, Experiment 2 suggests that these selective attention algorithms consist of feature tests to distinguish the two possible letters, rather than independent mechanisms specific to each of the individual letters.

A possible model of algorithm selection suggested by these conclusions is that subjects make a series of decisions about which algorithm to use and that each decision contributes to the total algorithm selection time. In order to predict a log-linear relationship between algorithm selection time and the number of alternative algorithms, it could be assumed that each decision, on the average, eliminated half of the possible algorithms. Thus, one decision would be required in the two-alternative condition, two in the four-alternative condition, and three in the eight-alternative condition. In this model, algorithm selection time increases with the number of alternative algorithms because the requisite number of algorithm decisions increases.

This paper has addressed an issue that is qualitatively different from the more traditional concerns of selective attention research. The latter can be characterized as investigating attentional capacities. Thus, attention research has investigated questions such as how many things one can attend to at once, what kinds of information can be ignored, and what kinds of information can be selectively attended to. In the present terms, these experiments are concerned with the kinds of selective attention algorithms that are possible. They say relatively little about the process of selecting such an algorithm.
One exception to this generalization is the study of "attention switching." In the present analysis, switching attention is accomplished by selecting a new attention algorithm. Attention switching time can be estimated in the split-span memory task (e.g., Broadbent, 1954), in which the subject is presented pairs of digits dichotically, one digit to each ear. Recall performance is typically better if subjects are allowed to report all of the digits on one channel first and then the digits on the other, rather than in the veridical temporal order. This difference in performance may be due to the extra time needed to switch attention back and forth between two channels (but see Gray \& Wedderburn, 1960; Moray, 1960; Penney, 1974; Yntema \& Trask, 1963). In this situation, attention switching time may be the time to select one of two algorithms differing in the source of sensory input (cf. Treisman, 1971). One algorithm would be used to process stimuli from the left ear, and the other would be used for stimuli from the right. Thus, the simplest thing for the subject to do would be to process the stimuli from one ear, switch algorithms once, and then process the stimuli from the other ear. A rough estimate of algorithm selection time can be obtained by comparing this ear-by-ear report order with a temporal report order, in which a new algorithm must be selected for each digit.

Other research is relevant to the process of algorithm selection if "algorithm" is interpreted in a larger sense. Since stimulus identification is only one part of the task confronting the subject, it may be reasonable to consider an attention algorithm as only one part of a larger algorithm used to perform the task as a whole. This task algorithm would include stimulus identification processes, decision processes, and response execution processes (Dixon, 1979). From this point of view, an algorithm for performing a choice reaction time task can be manipulated by varying possible responses as well as possible stimuli. Experiments varying algorithms in this way have found that algorithm selection time generally increases with the number of alternative algorithms, which is consistent with the present conclusions (Dixon \& Just, Note 1).

The algorithm selection effects found by Dixon and Just (Note 1) can be explained with a model similar to that proposed for the selection of stimulus identification algorithms. Algorithm selection would consist of a series of time-consuming decisions about components of the algorithm; generally more decisions would be needed when there were more alternative algorithms. However, Dixon and Just found that when algorithms differed only in terms of which finger was to be used for the response, no increase in algorithm selection time was found between two and four alternatives. This result can be explained by assuming that only one algorithm decision was required to choose which finger to use, regardless of how many fingers were possible in that condition. Thus, the two- and four-alternative conditions 
would both require only one decision (finger choice) and would have equal algorithm selection times. Using this assumption, algorithm selection in both the present experiments and those of Dixon and Just can be modeled as a series of time-consuming decisions.

In summary, this paper has taken the point of view that selective attention is controlled by an algorithm intentionally chosen prior to performing a task. The time to choose a selective attention algorithm for distinguishing two letters was measured in a trial initiation time paradigm. The results suggested that algorithms for distinguishing between letters may consist of perceptual tests for distinguishing features, and that the time to select such an algorithm is related to the number of alternatives. Although the present results are limited to one particular type of task, the paradigm seems to be general enough to be used to study the selection of algorithms in a variety of different attention tasks.

\section{REFERENCE NOTE}

1. Dixon, P., \& Just, M. A. Chronometric studies of algorithm selection: The problem of deciding what to do (Technical Memorandum 80-1222-1). Murray Hill, N.J : Bell Laboratories, 1980.

\section{REFERENCES}

Brainard, R. W., Irby, F. S., Fitts, P. M., \& Alluisi, E. A. Some variables influencing the rate of gain of information. Journal of Experimental Psychology, 1962, 63, 105-110.

Broadbent, D. E. The role of auditory localization in attention and memory span. Journal of Experimental Psychology, 1954, 47, $191-196$.

Dixon, P. Numerical comparison processes. Memory \& Cognition, $1978,6,454-461$.

Dixon, P. The selection and modification of algorithms in speeded tasks. Unpublished doctoral dissertation, CarnegieMellon University, 1979.

Ecreth, H. E. Selective attention. Psychological Bulletin, 1967, 67, 41-57.
FitTs, P. M. The information capacity of the human motor system in controlling the amplitude of movement. Journal of Experimental Psychology, 1954, 47, 381-391.

Fit's, P. M., \& Biederman, I. S-R compatibility and information reduction. Journal of Expperimental Psychology, 1965, 69, 408-412.

GARNER, W. R. Uncertainty and structure as psychological concepts. New York: Wiley, 1962.

Garner, W. R., \& Felfoldy, G. L. Integrality of stimulus dimensions in various types of information processing. Cognitive Psychology, 1970, 1, 225-241.

Gray, J. A., \& Wedderburn, A. A. I. Grouping strategies with simultaneous stimuli. Quarterly Journal of Experimental Psychology, 1960, 12, 180-189.

Нıск, W. E. On the rate of gain of information. Quarterly Journal of Experimental Psychology, 1952, 4, 11-26.

Moray, N. Broadbent's filter theory: Postulate $\mathrm{H}$ and the problem of switching time. Quarterly Journal of Experimental Psychology, 1960, 12, 214-220.

Morin, R. E., Forrin, B., \& Archer, W. Information processing: The role of irrelevant stimulus information. Journal of Experimental Psychology, 1961, 61, 89-96.

Penney, C. G. Dichotic listening and sequential associations in auditory short-term memory. Journal of Experimental Psychology, 1974, 102, 142-150.

Posner, M. I., \& Mitchell., R. A. A chronometric analysis of classification. Psychological Review, 1967, 74, 392-409.

Shepard, R. N., Kilpatric, D. W., \& Cunningham, J. P. The internal representation of numbers. Cognitive Psychology, 1975, 7, 82-138.

Treisman, A. M. Verbal cues, language, and meaning in attention. American Journal of Psychology, 1964, 77, 206-214.

Treisman, A. M. Strategies and models of selective attention. Psychological Review, 1969, 76, 282-299.

Treisman, A. M. Shifting attention between the ears. Quarterly Journal of Experimental Psychology, 1971, 23, 157-167.

YNTema, D. B., \& Trask, F. P. Recall as a search process. Journal of Verbal Learning and Verbal Behavior, 1963, 2, 65-74.

(Received for publication March 11, 1980; revision accepted July 30,1980 .) 